\title{
LE RÔLE DES FLUCTUATIONS FORTUITES DANS LA DIVERSITÉ DES POPULATIONS NATURELLES DE CEPAEA NEMORALIS (L.)
}

\author{
M. LAMOTTE \\ Laboratoire de Génétique de la Faculté des Sciences, Paris
}

Received 3.iv. 52

\section{INTRODUCTION}

L'ATTENTION a été attiré, depuis quelques années, sur un facteur longtemps négligé dans l'étude des phénomènes évolutifs: la variation fortuite des fréquences géniques, liée à l'effectif forcément limité des populations naturelles. S. Wright (I93 I, 1935) a montré, par des développements mathématiques, le rôle de cette "dérive génétique" ("genetic drift"), notamment dans l'évolution de populations de faible effectif. D'autres auteurs, au contraire, paraissent lui attribuer une bien moindre importance, sinon même lui dénier tout rôle, en regard des forces sélectives (Fisher et Ford, 1947; Cain et Sheppard, 1950). Mais bien peu d'exemples, au total, ont été donnés pour appuyer ces vues surtout théoriques, et leur interprétation prête encore souvent à discussion (Fisher et Ford, 1947; Wright, 1948). Ce sont en effet des centaines de générations qu'exigerait une expérimentation valable dans ce domaine, c'est-à-dire des dizaines d'anées au moins pour des animaux comme la Drosophile, dont le cycle vital est pourtant rapide.

Devant l'impossibilité de réaliser de telles expériences, nous avons cherché à transposer l'étude de la variation du plan temporel dans le plan spatial, en interprêtant les résultats fournis par l'examen simultané d'un ensemble de populations actuelles. Plus précisément, nous avons analysé les rapports existant entre le facteur qui est à la base même des fluctuations au hasard, la grandeur de l'effectif, et la diversité de composition génique des populations. Si cette diversité a pour origine des différences dans le niveau d'équilibre des gènès, différences qui peuvent d'ailleurs être liées à des facteurs internes-variété des compositions géniques globales des colonies-aussi bien qu'à des facteurs externes -actions divergentes du milieu environnant-, elle ne doit pas être influencée par la grandeur de l'effectif. Dans la mesure, au contraire, où interviennent des fluctuations fortuites, celles-ci auront un rôle d'autant plus marqué que l'effectif est plus faible: la diversité des fréquences géniques sera plus accentuée parmi les petites populations que parmi les grandes.

Une espèce nous a paru se prêter tout spécialement à une telle étude: l'Escargot des bois, Cepaea nemoralis (L.), qui présente le double 333 
avantage d'être polymorphe et de vivre en populations bien limitées (Lamotte, I951). Nous envisagerons plus particulièrement ici deux traits de ce polymorphisme dont le déterminisme génétique est connu (Lang, 1904, I909, I912; Lamotte, I95I): l'absence ou la présence de bandes pigmentées sur la coquille (gènes $b_{+}$et $b$ ) et la coloration rose ou jaune de cette coquille (gènes $j+$ et $j$ ).

\section{ETUDE EXPÉRIMENTALE DE L'INFLUENCE DE L'EFFECTIF SUR LA DISPERSION DES FRÉQUENCES GÉNIQUES}

Malgré les nombreuses recherches consacrées à l'interprétation des modalités du polymorphisme chez Cepaea nemoralis (Schilder, 1925; Boettger, I93 I Schnetter, 1950; Cain et Sheppard, i950), les données relatives à la composition génique des populations naturelles de l'espèce et, plus encore, à la grandeur de leur effectif, restaient encore très insuffisantes. De nombreuses colonies ont donc été étudiées dans toutes les régions de France, et leur composition déterminée à partir d'un échantillon aussi important que possible (Lamotte, 195I). Pour plusieurs centaines d'entre elles, on a pu également estimer l'effectif des escargots adultes, soit par la méthode des marquages et recaptures, soit par dénombrement direct des coquilles gisant sur le sol après brûlis des buissons où vivait la colonie, soit encore par comparaison avec des populations étudiées par l'une des deux méthodes précédentes. Nous considérerons ici plus particulièrement les deux catégories extrêmes de colonies: celles à effectif restreint-compris entre 500 et I000 individus-, et celles à effectif élevé-entre 3000 et 10.000 individus.

En ce qui concerne le gène inhibiteur de bandes $(b+)$, par exemple, les résultats suivants ont été observés (voir fig. I):

\begin{tabular}{|c|c|c|c|c|c|c|c|c|c|c|}
\hline $\begin{array}{l}\text { Fréquence } \\
\text { du gène } b+\end{array}$ & $\begin{array}{l}0, \\
-0,10\end{array}$ & $\begin{array}{l}0,10 \\
-0,20\end{array}$ & $\begin{array}{l}0,20 \\
-0,30\end{array}$ & $\begin{array}{l}0,30 \\
-0,40\end{array}$ & $\begin{array}{l}0,40 \\
-0,50\end{array}$ & $\begin{array}{l}0,50 \\
-0,60\end{array}$ & $\begin{array}{l}0,60 \\
-0,70\end{array}$ & $\begin{array}{l}0,70 \\
-0,80\end{array}$ & $\begin{array}{l}0,80 \\
-0,90\end{array}$ & $\begin{array}{c}0,90 \\
-1\end{array}$ \\
\hline $\begin{array}{l}\text { colonies de } \\
500 \text { à } 1000 \text { ind. }\end{array}$ & $\begin{array}{l}53 \\
(5)\end{array}$ & 23 & IO & 9 & 5 & 4 & 2 & - & - & $\begin{array}{l}3 \\
(3)\end{array}$ \\
\hline $\begin{array}{l}\text { colonies de } \\
3000 \text { à } 10.000 \text { ind. }\end{array}$ & $\begin{array}{l}46 \\
(5)\end{array}$ & I3 & II & 4 & I & I & I & - & - & - \\
\hline
\end{tabular}

Les dispersions de ces deux distributions sont mesurées respectivement par les variances*:

$$
\begin{aligned}
& V_{p}=35 \times 10^{-3} \\
& V_{g}=16 \times 10^{-3}
\end{aligned}
$$

c'est-à-dire que la dispersion des fréquences du gène "absence de bandes" est plus de deux fois supérieure dans les colonies de faible effectif.

* Les variances ont été calculées directement à partir des fréquences observées dans les populations, pour éviter l'inexactitude qu'introduirait le groupement. 

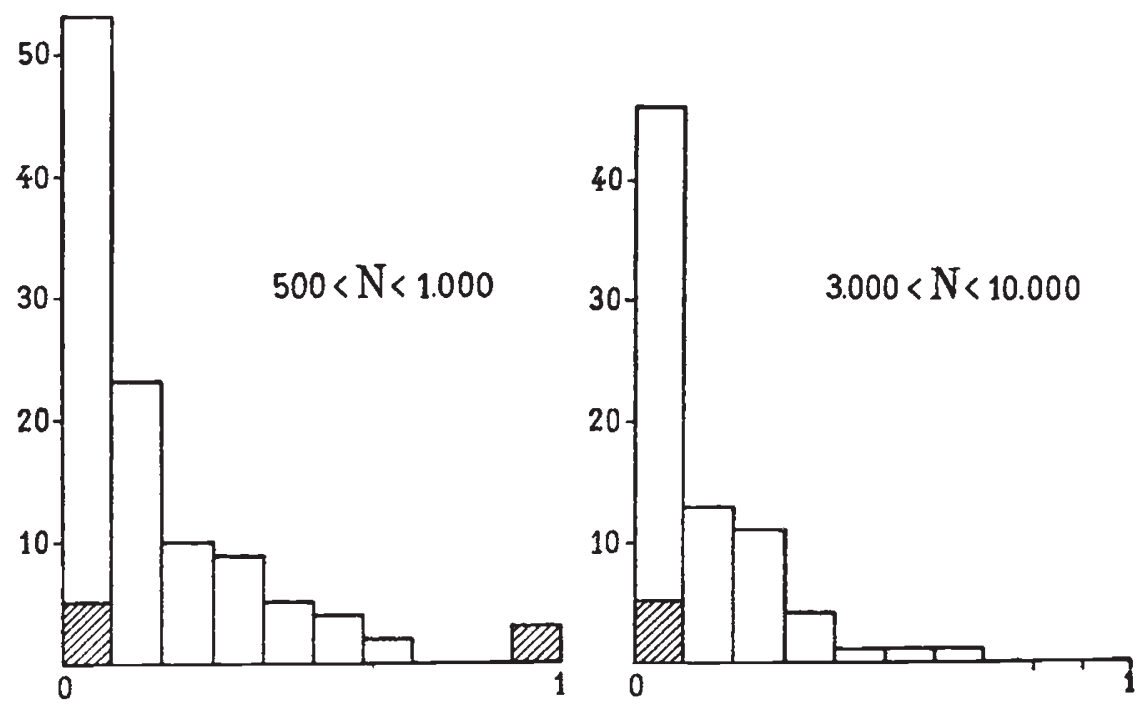

FIG. 1.-Distribution des fréquences du gène inhibiteur de bandes $\left(b_{+}\right)$dans les populations de faible et de fort effectif de Cepaea nemoralis.

Des résultats semblables se retrouvent dans le cas du gène $j$, déterminant la coloration jaune de la coquille (voir fig. 2):

\begin{tabular}{|c|c|c|c|c|c|c|c|c|c|c|}
\hline $\begin{array}{c}\text { Fréquence } \\
\text { du gène } \mathrm{j}\end{array}$ & $\begin{array}{l}0, \\
-0,10\end{array}$ & $\begin{array}{l}0,10 \\
-0,20\end{array}$ & $\begin{array}{c}0,20 \\
-0,30\end{array}$ & $\begin{array}{c}0,30 \\
-0,40\end{array}$ & $\begin{array}{l}0,40 \\
-0,50\end{array}$ & $\begin{array}{l}0,50 \\
-0,60\end{array}$ & $\begin{array}{l}0,60 \\
-0,70\end{array}$ & $\begin{array}{l}0,70 \\
-0,80\end{array}$ & $\begin{array}{c}0,80 \\
-0,90\end{array}$ & $\begin{array}{c}0,90 \\
-1\end{array}$ \\
\hline $\begin{array}{c}\text { colonies de } \\
500 \text { à 1000 ind. }\end{array}$ & $\begin{array}{c}6 \\
(2)\end{array}$ & 2 & 5 & 7 & 8 & 7 & 8 & 9 & $\begin{array}{c}7 \\
(5)\end{array}$ \\
\hline $\begin{array}{l}\text { colonies de } \\
\text { 3000 à 10.00o ind. }\end{array}$ & 0 & 3 & 4 & 6 & 5 & 7 & 6 & 5 & 5 & $\begin{array}{c}8 \\
(2)\end{array}$ \\
\hline
\end{tabular}

Les dispersions correspondantes sont:

$$
\begin{aligned}
& V_{p}=53 \times 10^{-3} \\
& V_{g}=29 \times 10^{-3}
\end{aligned}
$$
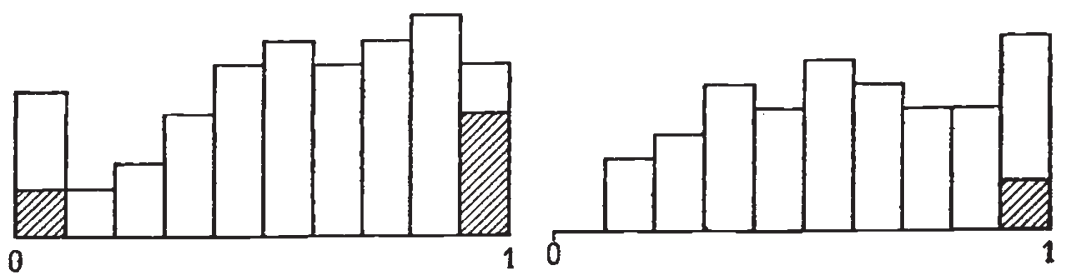

Fig. 2.-Distribution des fréquences du gène jaune ( $j$ ) dans les populations de faible et de fort effectif de Cepaea nemoralis. 
Ainsi, dans un cas comme dans l'autre, la dispersion de la distribution des fréquences du gène dans les populations de l'espèce diminue très nettement lorsque l'effectif s'accroît. Autrement dit, les petites colonies sont bien plus variées que les grandes.

A l'accroissement de la dispersion des fréquences géniques parmi les populations de faible effectif s'ajoute une autre caractéristique des distributions: la proportion plus grande des populations homogènes, où l'un des allèles a été éliminé. Ce nombre figure, en petits caractères, dans les classes marginales des quatre distributions précédemment étudiées. Pour le gène $b+$, par exemple, on constate que les trois colonies de faible effectif de la classe $0,90-1$ sont des colonies homogènes. Dans le cas du gène $j$, il n'existe que deux populations homogènes seulement dans la catégorie à effectif élevé, tandis qu'il y en a sept au totaldeux à fréquence nulle, cinq à fréquence 1 -parmi les colonies de faible effectif. La proportion des populations où l'un des allèles a été éliminé complètement est donc bien plus grande lorsque l'effectif est restreint.

\section{ETUDE THÉORIQUE DES RAPPORTS ENTRE L'EFFECTIF ET LES FLUCTUATIONS FORTUITES}

Ainsi, l'observation d'un grand nombre de populations permet de constater que la diminution de l'effectif va de pair avec un net accroissement de la dispersion des fréquences géniques, se manifestant par un nombre plus élevé de populations homogènes aussi bien que par l'augmentation de la variance de l'ensemble de la distribution. Ces faits ne peuvent guère s'expliquer que par l'intervention de fluctuations purement fortuites, dont ils sont la conséquence directe. En effet, si la fréquence d'un gène était, dans chaque colonie, déterminée strictement par l'ensemble des facteurs externes-les conditions du milieuet des facteurs internes-le patrimoine génétique global de la coloniel'effectif n'interviendrait en rien dans le niveau d'équilibre du gène, dont la distribution parmi les petites colonies serait, par conséquent, identique à sa distribution parmi les grandes colonies.

Il est intéressant de considérer la question sous son aspect quantitatif et de rechercher l'importance relative des fluctuations fortuites ainsi mises en évidence et des autres causes possibles de diversité des fréquences géniques, en particulier le rôle des facteurs externes, généralement mis en avant par les auteurs qui ont abordé déjà l'étude de Cepaea nemoralis (Schilder, r925; Boettger, 193I ; Cain et Sheppard, 1950).

La comparaison des variances des distributions apporte évidemment à la solution du problème une information quantitative précieuse. Mais il importe de remarquer que son interprétation n'est nullement immédiate: elle nécessite en effet de se reporter à un schéma théorique du comportement du gène considéré et d'y analyser le rôle de l'effectif.

Imaginons donc que les fluctuations fortuites constituent la seule cause de diversité des compositions géniques: on se trouve alors placé dans les conditions admises dans le schéma de S. Wright (193I), c'està-dire qu'il est possible, conaissant les taux de mutation de deux allèles 
et les taux de sélection des trois génotypes correspondants, de déduire la distribution des fréquences géniques dans les diverses populations de l'espèce, supposées toutes de même effectif. Il suffirait donc, en principe, de considérer le rapport des variances de " courbes de Wright" correspondant à des effectifs différents des colonies, toutes choses étant égales d'ailleurs : si ce rapport est égal à celui que fournissent les résultats expérimentaux, les fluctuations fortuites suffisent à expliquer la diversité observée; sinon, il faut admettre que des causes supplémentaires de diversité, que ne prévoient pas les hypothèses de Wright, viennent s'ajouter aux fluctuations fortuites, et leur importance sera d'autant plus grande que les rapports des variances différeront davantage.

Examinons d'abord un cas simplifié, où l'équilibre est déterminé par les seules mutations, directe et réverse, et par la migration, dont l'action peut être assimilée à celle de mutations (S. Wright, I93I). Dans ce cas, la relation de passage d'une génération à la suivante est linéaire et de la forme $\delta q=v-(u+v) q$, et la théorie de Wright conduit, pour l'état limite stationnaire de la distribution des fréquences, aux relations:

$$
\begin{aligned}
& \text { moyenne }=M=\frac{v}{u+v}, \text { indépendante de l'effectif } \mathcal{N} \text { des colonies; } \\
& \text { variance }=V=\frac{u v}{(u+v)^{2}} \quad \frac{\mathrm{I}}{4 \mathcal{N}(u+v)+\mathrm{I}}=M(\mathrm{I}-M) \frac{\mathrm{I}}{4 \mathcal{N}(u+v)+\mathrm{I}}
\end{aligned}
$$

Pour deux distributions correspondant à des colonies ne différant que par leurs effectifs $\mathcal{N}_{1}$ et $\mathcal{N}_{2}$, on a donc:

$$
\frac{V_{1}}{V_{2}}=\frac{4 \mathcal{N}_{2}(u+v)+\mathrm{I}}{4 \mathcal{N}_{1}(u+v)+\mathrm{I}}
$$

Lorsque les termes $4 \mathcal{N}(u+v)$ sont sensiblement supérieurs à I-ce qui est le cas pour des distributions "en cloche" -, on voit que la variance est à peu près inversement proportionnelle à l'effectif $\mathcal{N}$ des colonies, dans l'hypothèse faite où les fluctuations fortuites déterminent seules la diversité des fréquences géniques dans les populations de l'espèce.

Une telle comparaison analytique cesse malheureusement d'être possible dans le cas général où l'équilibre génique fait intervenir, outre les taux de mutation, des taux de sélection quelconques des trois génotypes en présence: ce qui est le cas, sans doute le plus fréquent, d'un polymorphisme "balancé", où l'hétérozygote possède une vitalité supérieure aux deux homozygotes. En effet, l'effectif intervient alors dans des relations transcendantes complexes dont on ne peut trouver de solution simple. On peut seulement affirmer que la variance se rapproche d'autant plus de zéro que l'effectif est plus grand.

Il est possible, toutefois, d'étudier numériquement le problème dans tous les cas particuliers que l'on désire, en construisant point par point chaque distribution théorique:

$$
f(q)=K q^{\mathbf{4} v-1}(1-q)^{4 N u-1} \exp .\left\{2 \mathcal{N} w q^{2}+4 \mathcal{N} t q\right\}
$$


et en déterminant graphiquement sa variance. Sans détailler les calculs, d'ailleurs fort laborieux, qui sont nécessaires, nous nous bornerons à exposer ici les résultats obtenus pour un certain nombre de valeurs des paramètres relatives à des courbes de Wright qui se rapprochent sensiblement de celles observées expérimentalement.

Les paramètres adoptés en premier lieu sont les meilleures estimations correspondant à la distribution du gène $\mathrm{b}+$ réellement observée dans 280 populations naturelles d'Aquitaine (Lamotte, I95I): les taux de mutation et de sélection $u, v, w$ et $t^{*}$ sont égaux respectivement à $u=24 \times \mathrm{IO}^{-3}, v=0,6 \times \mathrm{IO}^{-3}, w=44 \times \mathrm{IO}^{-3}$ et $t=\mathrm{I} 8 \times \mathrm{IO}^{-3}$. On a pris successivement pour l'effectif des colonies les valeurs 500, 1000, 2000, 4000 et 8000 ; ce sont les courbes théoriques ainsi définies que reproduit la figure $3 a$.

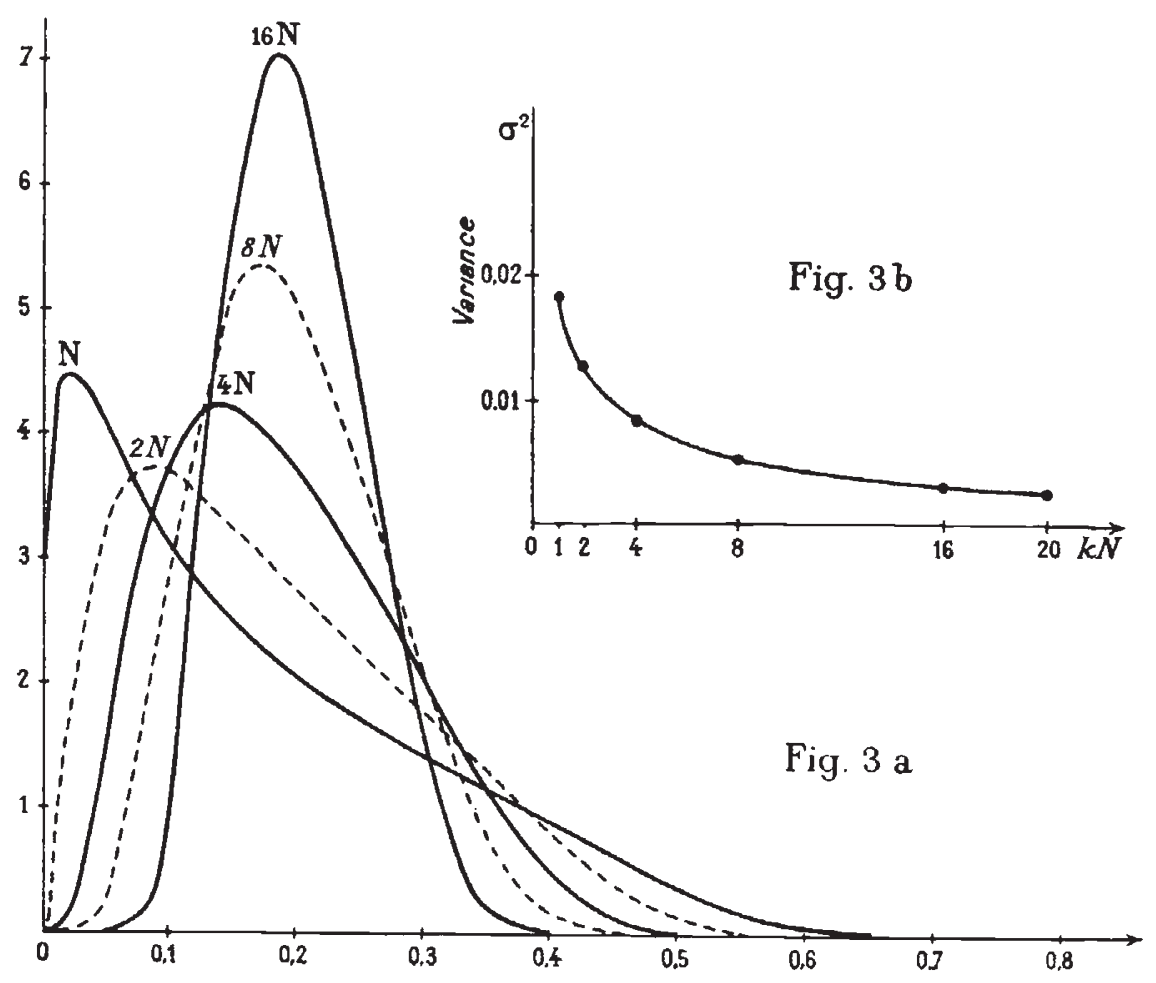

Fig. 3A.-Courbes de Wright correspondant aux paramètres de mutation et de sélection $\mathrm{u}=24 \times 10^{-8}, \mathrm{v}=0,6 \times 10^{-8}, \mathrm{w}=44 \times 10^{-3}, \mathrm{t}=18 \times 10^{-3}$, et aux différents effectifs $\mathrm{N}=500,2 \mathrm{~N}=1000,4 \mathrm{~N}=2000,8 \mathrm{~N}=4000$ et $16 \mathrm{~N}=8000$.

FIG. 3B.-Loi de variation, en fonction de l'effectif, des variances des courbes de Wright correspondant aux paramètres de 3 a.

* Voir Malécot (r948) et Lamotte (1951). Les paramètres u et v traduisent les effets conjoints de la mutation et de la migration.

Quant aux paramètres de sélection $w$ et $t$, introduits par Malécot, ils sont définis par les expressions $\mathrm{w}=\frac{\sigma_{1}-2 \sigma_{2}+\sigma_{3}}{\sigma_{1}}$ et $\mathrm{t}=\frac{\sigma_{2}-\sigma_{1}}{\sigma_{1}}$, où $\sigma_{1}, \sigma_{2}$ et $\sigma_{3}$ sont les valeurs sélectives respectives de l'homozygote récessif, de l'hétérozygote et de l'homozygote dominant. 
Le tableau qui suit indique les valeurs des variances de ces distributions, dont la figure $3^{b}$ représente graphiquement la loi de variation en fonction de l'effectif des colonies.

$\begin{array}{lccccc}\text { Effectif } & 500 & 1000 & 2000 & 4000 & 8000 \\ \text { Variance } & 18,5 & 12,9 & 8,4 & 5,2 & 3,1 \\ & \times 10^{-3} & \times 10^{-3} & \times 10^{-3} & \times 10^{-3} & \times 10^{-3}\end{array}$

Deux autres familles de distributions théoriques ont été étudićes, dont chacune correspond à des valeurs déterminées des paramètres de mutation et de sélection et, comme précédemment, aux divers effectifs 500, 1000, .. 8000.

La première de ces deux séries a pour paramètres: $u=5 \times 10^{-3}$, $v=0,5 \times 10^{-3}, w=0$ et $t=5 \times 10^{-3}$. On trouve, pour les variances des diverses distributions:

$\begin{array}{lccccc}\text { Effectif } & 500 & 1000 & 2000 & 4000 & 8000 \\ \text { Variance } & 23,5 & 13,5 & 7,3 & 3,8 & 2,0 \\ & \times 10^{-3} & \times 10^{-3} & \times 10^{-3} & \times 10^{-3} & \times 10^{-3}\end{array}$

Pour la seconde série, les paramètres sont: $u=5 \times 10^{-3}, v=0,5 \times 10^{-3}$ $w=0$ et $t=2,5 \times 10^{-3}$. Les variances trouvées, correspondant aux divers effectifs sont alors:

$\begin{array}{lccccc}\text { Effectif } & 500 & 1000 & 2000 & 4000 & 8000 \\ \text { Variance } & 13,1 & 7,6 & 4,2 & 2,3 & 1,4 \\ & \times 10^{-3} & \times 10^{-3} & \times 10^{-3} & \times 10^{-3} & \times 10^{-3}\end{array}$

Ces quelques applications numériques à des distributions théoriques de S. Wright donnent une idée de la décroissance de la variance de ces distributions lorsqu'augmente l'effectif des populations. Elles ne permettent pas de déterminer l'expression d'une loi analytique commune, qui dépend d'ailleurs des divers paramètres, mais il apparait que, dans les cas étudiés, la variance décroît plus lentement que l'inverse de l'effectif. Plus précisément, ces résultats montrent que le rapport des variances correspondant aux effectifs $\mathcal{N}_{g}$ (400o individus en moyenne) et $\mathcal{N}_{p}$ (entre 500 et 1000 individus) des deux groupes de colonies de C.n. étudiés plus haut, est compris entre $\mathrm{r} / 3$ et $\mathrm{r} / 5$; quant à la variance de la distribution des colonies de fort effectif $\left(\mathcal{N}_{g}\right)$, on voit qu'elle est comprise entre le quart et la moitié de la différence des variances des deux groupes.

\section{INTERPRÉTATION DES RÉSULTATS OBSERVÉS}

Revenons maintenant aux résultats observés pour les gènes " jaune" et "absence de bandes" dans les populations de Cepaea nemoralis.

Pour le gène "jaune", tout d'abord, nous avons vu que la variance passait de $53 \times 10^{-3}$ dans la distribution des petites colonies à $29 \times 10^{-3}$ dans celle des grandes, dont l'effectif est, en moyenne, quatre à six fois plus élevé. Or, d'après les lois théoriques qui viennent d'être exposées, le rapport de ces variances devrait être notablement plus fort et dépasser 3 ou 4: il faut en conclure que les fluctuations fortuites ne sont pas seules à l'origine de la diversité des fréquences géniques et que d'autres causes, indépendantes de l'effectif des populations, interviennent également. On peut remarquer, d'ailleurs, que les variances sont anormale- 
ment élevées pour être simplement dues à des fluctuations fortuites. Mais il n'en reste pas moins que, dans le cas des petites colonies, d'effectif inférieur à un millier d'individus, la diversité due aux fluctuations fortuites doit représenter, mesurée par la variance des distributions, la moitié au moins de la diversité globale, puisqu'elle diminue de moitié lorsque l'effectif s'accroît.

Chez les populations d'effectif élevé, la part de la dérive génétique dans la diversité observée ne peut plus être évaluée directement comme ci-dessus, puisqu'il serait nécessaire de considérer la dispersion génique parmi des populations d'effectif infini, qui n'existent évidemment pas. L'examen des lois théoriques de variation de la variance en fonction de l'effectif permet toutefois d'en rechercher une approximation.

Nous avons remarqué, en effet, que la variance fortuite donnée par les formules de Wright était comprise, pour les colonies d'effectif voisin de 4000 , entre le quart et la moitié de la différence des variances relatives aux distributions des grandes et des petites colonies. On peut donc estimer que la part de la dispersion génique qui revient aux fluctuàtions fortuites, dans le cas des grandes colonies, correspond à une variance supérieure à $\frac{53 \times \mathrm{IO}^{-3}-29 \times \mathrm{IO}^{-3}}{4}=6 \times \mathrm{IO}^{-3}$. Comme la variance totale est $29 \times \mathrm{IO}^{-3}$, la part des fluctuations fortuites en représente au moins le cinquième.

Dans le cas des petites colonies, la part des fluctuations fortuites dépasse donc $\left(53 \times \mathrm{IO}^{-3}-29 \times \mathrm{IO}^{-3}\right)+6 \times \mathrm{IO}^{-3}$ soit $30 \times \mathrm{IO}^{-3}$, c'est-àdire plus de la moitié de la variance globale.

Le reste de la diversité semble devoir être attribué à l'action du milieu, différent selon les biotopes, comme nous aurons l'occasion de la montrer ultérieurement.

Dans le cas du gène " absence de bandes", la variance de la distribution des petites colonies est sensiblement plus de deux fois supérieure à celle de la distribution des grandes: $35 \times 1 \mathrm{IO}^{-3}$ au lieu de $\mathrm{I} 6 \times \mathrm{IO}^{-3}$. Le rôle des fluctuations fortuites est donc plus important encore que précédemment.

Pour les populations d'effectif élevé, la part de la dispersion imputable à ces fluctuations peut être estimée, de la même façon que pour le gène "jaune", au quart au moins de la différence entre les variances des distributions des petites et des grandes colonies, soit ici $\frac{35 \times 10^{-3}-16 \times 10^{-3}}{4}=5 \times 10^{-3}$. Pour les petites colonies, la dispersion due aux fluctuations fortuites dépassera donc $\left(35 \times 10^{-3}-16 \times \mathrm{IO}^{-3}\right)$ $+5 \times 10^{-3}=24 \times 10^{-3}$, c'est-à-dire les deux tiers de la variance totale qui est $35 \times 10^{-3}$.

Il convient même de remarquer que la part de la dérive génétique apparaîtrait relativement bien plus forte encore dans le cadre d'une région limitée et non plus dans l'ensemble de toutes les régions de France. Il existe en effet des divergences régionales importantes dans 
la fréquence moyenne d'équilibre du gène "absence de bandes" (Lamotte, I95I). Ces divergences sont sans rapport aucun avec les facteurs externes, climatiques ou autres, et dues à l'isolement relatif des populations: c'est le faible pouvoir de déplacement des individus qui a amené la ségrégation de races locales élémentaires, différant par le niveau moyen d'équilibre des principaux gènes du polymorphisme. Une telle variabilité locale doit naturellement accroître de façon identique la dispersion des fréquences géniques parmi les grandes et parmi les petites colonies, qui proviennent les unes et les autres de l'ensemble des provinces.

Une étude faite dans une sule région aurait permis, en éliminant ce facteur régional de diversité, de confronter seulement les rôles respectifs des fluctuations fortuites et des différences d'équilibre sélectif liées à la diversité des biotopes; la nécessité de considérer un nombre de populations suffisamment élevé n'a pas permis de la réaliser. Il est toutefois possible d'estimer la part qui, dans la diversité génique de l'ensemble des colonies, revient à l'hétérogénéité des stocks régionaux: il suffit de déterminer la variance de la distribution des fréquences géniques régionales. Ces fréquences moyennes* du gène " absence de bandes" sont, dans les principales régions d'où proviennent les populations étudiées:

\begin{tabular}{|c|c|c|c|c|c|}
\hline Aquitaine & - & - 0,19 & Somme & - & - 0,16 \\
\hline Pyrénées & - & $-0,15$ & Alpes - & - & $-0,17$ \\
\hline Bretagne & - & $-0,16$ & Centre & - & $-0,11$ \\
\hline Région Paris & & $-0,12$ & Rég. Rhod. & - & - 0,32 \\
\hline Nord & - & - 0,07 & Mass. Cientr. & & $-0,09$ \\
\hline Est & - & - 0,29 & Ouest & - & $-0,11$ \\
\hline
\end{tabular}

d'où la variance d'hétérogénéité régionale:

$$
V_{\text {het }}=6 \times \mathrm{IO}^{-3}
$$

On peut donc estimer que, dans le cadre d'une région limitée, les dispersions du gène " absence de bandes" seraient respectivement, dans les petites et dans les grandes colonies†:

$$
\begin{aligned}
& V_{p}=35 \times 10^{-3}-6 \times 10^{-3}=29 \times 10^{-3} \\
& V_{g}=16 \times 10^{-3}-6 \times 10^{-3}=10 \times 10^{-3}
\end{aligned}
$$

Dans ces conditions, la part de variance revenant aux fluctuations fortuites, que nous avons trouvée égale à $24 \times \mathrm{IO}^{-3}$ pour les colonies de faible effectif et à $5 \times 10^{-3}$ pour les colonies d'effectif élevé, représente, pour les premières, les $5 / 6$ de la variance totale et, pour les autres, elle en est encore la moitié.

\section{CONCLUSIONS}

Les fluctuations fortuites-ou dérive génétique--sont donc à l'origine d'une part importante de la diversité que l'on observe parmi les

* Dont chacune a été déterminée à partir de plusieurs dizaines de colonies.

† Une bonne confirmation en est apportée par les valeurs des variances réellement observées pour l'ensemble des colonies, de tous effectifs, dans chacune des régions : ces variances sont en effet comprises entre les deux valeurs précitées. En Aquitaine, par exemple, $\mathrm{V}=13 \times 10^{-3}$; dans la région parisienne, $\mathrm{V}=15 \times 10^{-3}$; dans les Pyrénées, $\mathrm{V}=14 \times 10^{-3}$; en Bretagne, $\mathrm{V}=20 \times 10^{-3}$. 
fréquences géniques dans les populations naturelles de C.n. Il faut voir là, sans doute, une conséquence directe de l'effectif en général très limité des colonies de cette espèce, et cela d'autant plus que l' " effectif efficace" (" effective size") est certainement bien plus réduit encore: l'instabilité des biotopes habituels de l'espèce-biotopes " secondaires", liés à l'action de l'homme-limite en effet la durée d'existence de la plupart des colonies et accroît par là l'importance relative des premières années de la colonie, où l'effectif est naturellement très restreint et où, par conséquent, les fluctuations au hasard peuvent jouer un rôle primordial.

Mais il faut considérer aussi ce rôle important de la dérive génétique dans la distribution des fréquences de certains gènes-et c'est plus particulièrement le cas, ici, du gène " absence de bandes"-comme la marque d'un équilibre génique déterminé sans grande rigueur, c'està-dire lié à des valeurs sélectives de très faible intensité.

Il serait évidemment hasardeux de vouloir généraliser de telles conclusions à n'importe quel autre gène et, plus encore, à n'importe quelle autre espèce, de même qu'il n'est guère admissible de dénier systématiquement tout rôle aux fluctuations fortuites, dont l'existence est une conséquence inéluctable de l'effectif limité des populations naturelles. Il est évident que chaque espèce et, dans chacune d'elle, chaque gène, constitue un cas particulier qu'il convient d'étudier en détail si l'on veut pouvoir conclure à son endroit: la comparaison des cas respectifs du gène "jaune" et du gène " absence de bandes " montre bien cette diversité de comportement à l'intérieur d'une même espèce et l'examen d'autres gènes ne ferait que confirmer ce point de vue.

D'une façon très générale, la part de la dérive génétique sera d'autant plus importante dans la dispersion des fréquences d'un gène que les niveaux d'équilibre géniques seront moins strictement déterminés. Dans le cas, assez rare sans doute dans la nature, où n'existe, dans les diverses populations de l'espèce, qu'un seul niveau d'équilibre pour un gène déterminé-c'est-à-dire, pratiquement, lorsque cet équilibre est indépendant des conditions de milieu-, les distributions théoriques de $\mathrm{S}$. Wright donnent directement une image quantitative du phénomène. Dans les autres cas, elles permettent de rechercher une estimation des rôles des différents facteurs de diversité, ainsi qu'il a été fait pour C.n.

\section{ENGLISH SUMMARY}

The polymorphism of Cepaea nemoralis (L.) is characterised by an extreme variation in the frequencies of varieties in different natural populations. The author has set out to determine the role of fortuitous fluctuations (genetic drift) in this diversity.

Toward this end, a comparative study was made of the scatter of the frequencies of genes $b+$ and $j$ in two categories of colonies: those composed of many individuals (over 300o) and those composed of few (less than 1000). If the gene frequencies were in all instances rigorously determined by the interplay of selective forces, their distribution would 
be independent of the number of individuals in the colonies. In fact, this proves not to be the case, for variation is considerably greater among the small populations than it is among the big. Thus, genetic drift must play an important role in the diversity of the gene frequencies of these populations, for it is the only factor which depends upon population size.

The author has tried to determine quantitatively the portion of variation which is due to genetic drift, in view of the difference in the values of genic equilibria in the various environments. In order to do this, a study was made of the expected variation of the scatter as a function of population size, assuming that genetic drift alone operated. This was done by determining the variance of Wright's curves corresponding to different colony sizes. The relationship of the variances actually observed in the two categories of colonies was compared with the theoretical law of variation thus estimated.

It appears that genetic drift has a less appreciable role in determining variation of yellow and pink forms, whereas its role is preponderant in determining variation of banded and unbanded forms. In the case of the latter, even in large colonies genetic drift is responsible for half of the scatter observed within a given limited region. In small colonies, which are the most frequent, fluctuations resulting from genetic drift represent $5 / 6$ of the total scatter.

\section{REFERENCES}

Boettger, c. I93I. Die Entstehung von Populationen mit bestimmter Variantenzahl bei der Landschneckengattung Cepaea. Z.I.A.V., 58, 295-345.

CAIN, A. J., AND SHEPPARD, P. M. I950. Selection in the polymorphic land-snail Cepaea nemoralis. Heredity, 4, 275-294.

DIVER, C. 1939. Aspects of the study of variation in snails. $\mathcal{F}$. of Conchol., 21, 9I-I4I. FISHER, R. A., AND FORD, E. B. 1947. The spread of a gene in a colony of the moth Panaxia dominula. Heredity, 1 , I 43-1 74 .

LAMOTTE, M. I95I. Recherches sur la structure génétique des populations naturelles de Cepaea nemoralis. Bull. Biol., Suppl. 35, I-238.

LANG, A. 1904. Über Vorversuche zu Untersuchungen über die Varietätenbildung von $H$. hortensis und $H$. nemoralis. Festschrift $f$. Haeckel. Fischer, Iena.

LANG, A. Igog. Über Vererbungsversuche. Verh. d. deutsch. Zool. Gesselsch., 19, i 7-80.

LANG, A. I9I2. Vererbungswissenschaftliche Miszellen. Z.I.A.V., 8, 233-283.

MALÉCOT, C. 1948. Les mathématiques de l'hérédité. Masson et Cie., Paris.

SCHILder, F. A. 1925. Zur Variabilität von Cepaea. Z.I.A.V., 39, 249-28o.

SCHILder, F. A. 1950. Die Ursachen der Variabilität bei Cepaea. Biol. Zbl., 69, 79-1 03.

SCHNETTER, M. I950. Veränderungen der genetischen Konstitution in naturlichen Populationem der polymorphen Bänderschnecken. Verh. d. deutsch. Zool. in Marburg, 13, I92-206.

WRIGHT, s. I93 I. Evolution in mendelian populations. Genetics, 16, 97-159.

WRIGHT, s. 1935. Evolution in populations in approximate equilibrium. $\mathscr{f}$. Genetics, $30,257-266$.

WRIGHT, s. 1948. On the roles of directed and random changes in gene frequency in the genetics of populations. Evolution, 2, 279-294. 